\title{
Cortical organization of inhibition-related functions and modulation by psychopathology
}

\section{Stacie L. Warren ${ }^{1,2}{ }^{*}$, Laura D. Crocker ${ }^{1}$, Jeffery M. Spielberg ${ }^{1,3}$, Anna S. Engels ${ }^{1,4}$, Marie T. Banich ${ }^{5}$, Bradley P. Sutton ${ }^{6}$, Gregory A. Miller ${ }^{1,78}$ and Wendy Heller ${ }^{1}$}

${ }^{1}$ Department of Psychology, University of Illinois at Urbana-Champaign, Champaign, IL, USA

2 Department of Mental Health, St. Louis VA Medical Center, St. Louis, MO, USA

${ }^{3}$ School of Public Health, University of California, Berkeley, Berkeley, CA, USA

${ }^{4}$ Department of Psychology, Pennsylvania State University, University Park, PA, USA

${ }^{5}$ Department of Psychology, University of Colorado at Boulder, Boulder, CO, USA

${ }^{6}$ Department of Bioengineering, University of Illinois at Urbana-Champaign, Urbana, IL, USA

7 Department of Psychology, University of Delaware, Newark, DE, USA

${ }^{8}$ Department of Psychology, University of Konstanz, Konstanz, Germany

\section{Edited by:}

Alexander J. Shackman, University of Maryland, USA

\section{Reviewed by:}

Daniel Grupe, Waisman Lab for Brain Imaging and Behavior, USA

Alan Anticevic, Yale University, USA

\section{*Correspondence:}

Stacie L. Warren, Department of Mental Health, St. Louis VA Medical Center, \#1 Jefferson Barracks Drive, (116B/JB), St. Louis, MO 63125, USA

e-mail: staciewarren3@gmail.com
Individual differences in inhibition-related functions have been implicated as risk factors for a broad range of psychopathology, including anxiety and depression. Delineating neural mechanisms of distinct inhibition-related functions may clarify their role in the development and maintenance of psychopathology. The present study tested the hypothesis that activity in common and distinct brain regions would be associated with an ecologically sensitive, self-report measure of inhibition and a laboratory performance measure of prepotent response inhibition. Results indicated that sub-regions of DLPFC distinguished measures of inhibition, whereas left inferior frontal gyrus and bilateral inferior parietal cortex were associated with both types of inhibition. Additionally, co-occurring anxiety and depression modulated neural activity in select brain regions associated with response inhibition. Results imply that specific combinations of anxiety and depression dimensions are associated with failure to implement top-down attentional control as reflected in inefficient recruitment of posterior DLPFC and increased activation in regions associated with threat (MTG) and worry (BA10). Present findings elucidate possible neural mechanisms of interference that could help explain executive control deficits in psychopathology.

Keywords: inhibition, anxiety, depression, DLPFC, attentional control

\section{INTRODUCTION}

Despite a lack of consensus on how best to define executive function (EF), neuropsychological and neuroimaging (Collette et al., 2005) research indicates that EF may be usefully characterized as a collection of correlated yet dissociable processes: inhibition, set shifting, and working memory updating (e.g., Miyake et al., 2000). Inhibition-related processes in particular are considered to be critical for top-down cognitive control and its translation to real-word, everyday behavior, including self-regulation and emotion regulation (Zelazo and Cunningham, 2007). Further, inhibition-related functions are essential for efficient working memory function, limiting access to and removing information that is no longer necessary (Friedman and Miyake, 2004). Cognitive disruptions in these processes are a prominent source of distress and impairment and have been implicated in anxiety and depression (Eysenck et al., 2007; Levin et al., 2007; Joormann and Gotlib, 2010; Snyder, 2013; Warren et al., under review). To the degree that the experience of negative mood states and negative life events activates mood-congruent representations in working memory (Siemer, 2005), identifying specific inhibitionrelated dysfunctions associated with anxiety and depression could lead to relatively specific targets for intervention.
Not only do inhibition-related processes contribute to aspects of daily life, they play a critical role in psychopathology, as deficits in these processes have been implicated in the affective and cognitive symptoms of anxiety and depression. In particular, intrusive thoughts such as worry and rumination are hallmark characteristics of anxiety and depression, respectively, and several researchers have suggested that these symptoms are a result of impaired inhibition (Hertel, 1997; Eysenck et al., 2007; Joormann, 2010; see Anticevic et al., 2012, and Fox et al., 2012, for potential contributions of neural networks to psychopathology symptoms). Anxiety has been associated with broad impairments in attentional control, including increased distractibility and impaired processing efficiency (e.g., resource utilization) as opposed to performance effectiveness (e.g., percentage of correct responses; Eysenck et al., 2007; Eysenck and Derakshan, 2011). Research in depression has repeatedly demonstrated problems with attention, memory, and problem-solving abilities (Yee and Miller, 1994; Weiland-Fiedler et al., 2004; Levin et al., 2007; Warren et al., 2008), and impaired inhibition is hypothesized to facilitate these cognitive disruptions via effects on working memory (e.g., Joormann and Gotlib, 2010). Thus, making an explicit link among individual differences in specific inhibition-related functions and dimensions 
of anxiety and depression is important for understanding the intricate relationship between affective experiences and cognitive control.

Colloquially, the term inhibition is used with respect to control of behaviors in everyday life (e.g., distraction, impulsivity), although the contribution of specific inhibition-related functions is not well understood. Notably, most formal tests of EF were developed and administered in understandably artificial environments (e.g., laboratory or controlled testing environment). Although research is advancing in determining the cognitive processes that these formal tests of EF actually measure (e.g., Miyake et al., 2000), the degree to which activities of daily life require these same processes is unclear (Burgess et al., 2009). The present study sought to identify empirically specific neural mechanisms implementing the type of inhibition that has been demonstrated clearly in a laboratory setting, (e.g., prepotent response inhibition) as well as behavioral inhibition measured in everyday life. Given that impaired inhibition-related functions have been implicated as risk factors for a broad range of psychopathology, it is important that the nature of inhibition-related processes be specified.

Individual differences in specific inhibition-related functions at the level of neural mechanisms might be more strongly tied to the development and maintenance of psychopathology than the broader construct of inhibition as a whole. Neuroimaging studies exploring inhibition have demonstrated the involvement of various regions, including dorsolateral prefrontal cortex (DLPFC), inferior frontal gyrus (IFG), and anterior cingulate cortex (ACC), although lesion studies implicate right IFG in particular (see Aron et al., 2004, for a review). DLPFC, ACC, and IFG appear to facilitate task performance in inhibition paradigms. However, it is likely that inhibition co-exists with other cognitive functions required by these tasks (e.g., updating, shifting), making it difficult to determine which brain regions are involved in the implementation of specific inhibition processes. DLFPC is associated with top-down attentional control (e.g., Dosenbach et al., 2008), maintaining goals, and updating information (e.g., Wager and Smith, 2003), whereas ACC is involved in detecting response conflict and monitoring performance (Nelson et al., 2003; Banich et al., 2009). IFG is activated when an individual needs to resolve interference among potentially conflicting attributes of stimuli (Nelson et al., 2003; for review of left IFG, see Jonides and Nee, 2006) and may function to inhibit incorrect responses (Aron et al., 2004). Further, IFG appears to play a more general role in responding to salient, task-related cues as part of an EF network (Hampshire et al., 2010).

Although there is some support for inhibitory dysfunction in both anxiety and depression, the literature to date is mixed (Derakshan and Eysenck, 2009; Snyder, 2013; Snyder et al., under review). Several methodological and conceptual issues could account for discrepant results. Cognitive tasks that are commonly employed often each rely on multiple aspects of EF that might be impaired in psychopathology, making it difficult to draw firm conclusions about the presence of inhibition-related deficits specifically (Henry and Crawford, 2005). In addition, the concept of "inhibition" is broad, and tasks that are assumed to measure inhibition vary in their definition and implementation, making it difficult to ascertain the nature of the function measured. Finally, evidence suggests that co-occurring disorders may have both additive and interactive effects on brain activity and EF (Keller et al., 2000; Moritz et al., 2001; Basso et al., 2007; Engels et al., 2010; Herrington et al., 2010) as well as on clinical outcomes (e.g., Emmanuel et al., 1998). Yet many studies fail to assess or control comorbidity, making it difficult to parse the effects of specific dimensions of anxiety and depression on inhibition impairments and related brain activity.

Depression is distinguishable from two types of anxiety, anxious apprehension and anxious arousal (Heller et al., 1997; Nitschke et al., 1999, 2001). Anxious apprehension is characterized by worry and verbal rumination (Andrews and Borkovec, 1988; Barlow, 1991, 2002), whereas anxious arousal is characterized by somatic tension and sympathetic hyperarousal (Watson et al., 1995a,b). In contrast, depression is characterized by decreased responsivity to pleasurable stimuli (i.e., anhedonia; APA, 2000) and low positive affect (Watson et al., 1995a).

Hemodynamic neuroimaging studies of anxiety and depression have identified abnormal function in regions associated with inhibition-related processes, including prefrontal cortex (particularly DLPFC and IFG), ACC, and areas within parietal cortex (Mayberg, 1997; Mayberg et al., 1999; Rogers et al., 2004; Pizzagalli et al., 2006; Engels et al., 2007, 2010; Herrington et al., 2010). Further, when distinctions between depression and types of anxiety are taken into account, distinct patterns of neural activity emerge. For example, Engels et al. (2007, 2010) demonstrated that anxious apprehension is associated with increased left IFG (Broca's area) activity, whereas anxious arousal is associated with increased right inferior temporal gyrus (ITG) activity. Herrington et al. (2010; see also Miller et al., 2013) demonstrated that depression is associated with rightward lateralization of DLPFC activity. Given that individual differences in inhibitionrelated functions have been implicated as risk factors for anxiety and depression, a second goal of the present study is to examine how these dimensions of psychopathology (anxious apprehension, anxious arousal, and anhedonic depression) modulate neural mechanisms supporting specific inhibition-related functions. Understanding these relationships could contribute to an account of psychological or neural mechanisms involved in the development and maintenance of symptoms of psychopathology, as well as inform current and potential methods of treatment targeting the cognitive biases and impairments associated with anxiety and depression.

Based on the review above, it was hypothesized that regions involved in a frontal-parietal network supporting inhibitionrelated processes will be associated with both self-reported behavioral inhibition in everyday life and prepotent response inhibition. In addition, it was anticipated that distinct neural mechanisms would be associated with the two aspects of inhibition under investigation. It was hypothesized that Stroop reaction time (RT) interference, a measure of prepotent response inhibition that likely reflects greater active suppression than selfreported inhibition in everyday life, would be associated with DLPFC, ACC, and IFG activity. These regions have been implicated in implementing cognitive control, as well as response inhibition (Banich et al., 2000; Milham and Banich, 2005; Banich, 
2009). In particular, it was anticipated that RT interference would be associated with posterior DLPFC activity, as this region is considered to be critically involved in performance on the Stroop task, in part by biasing other brain regions toward processing task-relevant information (e.g., color of the ink) and away from task-irrelevant information (e.g., reading the color word). Thus, posterior DLPFC is thought to be particularly involved in implementing resistance to a dominant response. In contrast, it was hypothesized that self-reported behavioral inhibition would be associated with mid-DLPFC activity, as this region is implicated in multitasking functions and responding to context (Crocker et al., 2012), as well as maintaining task-relevant information (Kane and Engle, 2002; Banich, 2009). Thus, mid-DLPFC is associated with resisting distraction. Further, given mid-DLPFC's role in maintaining task-relevant information and resisting distraction, it was anticipated that worse self-reported inhibition (e.g., impulsivity, distractibility) would be associated with increased activity in this area. Given that response-inhibition paradigms have dominated much of the inhibition neuroimaging literature, it is unknown whether self-reported inhibition as measured in everyday life will elicit IFG and ACC activity. To the degree that self-reported inhibition relies on stopping behavioral responses, it is likely to be associated with IFG activation. A correlation with ACC may be less likely, as this region is recruited during tasks that generate conflicting, response-related representations, such as the incongruent condition of a Stroop task ("RED" printed in blue ink; Banich, 2009).

Further, given empirical support from hemodynamic neuroimaging studies that have properly accounted for comorbidity between depression and anxiety or comorbidity among anxiety types (Engels et al., 2007, 2010; Herrington et al., 2010), it was hypothesized that depression and anxiety would be associated with opposing effects on inhibition-related brain activity. For both prepotent response inhibition and self-reported inhibition in everyday life, it was anticipated that depression would be associated with decreased left DLPFC and ACC activity. It was also hypothesized that depression would be associated with decreased posterior DLPFC response inhibition activity, as previous work has shown hypoactivation in this area (e.g., Herrington et al., 2010). In contrast, anxiety should be accompanied by greater activation in brain areas associated with attentional control in distracting conditions (see Eysenck and Derakshan, 2011, for review). It was expected that anxiety of either type (anxious apprehension and anxious arousal) would increase activity in mid-DLPFC associated with self-reported inhibition, activity in posterior DLPFC associated with response inhibition, and ACC activity associated with both measures of inhibition, as these regions have been shown to play prominent roles in attentional control (e.g., Engels et al., 2007, 2010; Banich, 2009). It was also anticipated that anxious apprehension would increase left IFG activity associated with response inhibition, as previous work has shown hyperactivation in this area (Engels et al., 2007).

\section{MATERIALS AND METHODS PARTICIPANTS}

Eighty-five paid undergraduate participants ( 52 females, age $M=$ $19.08, S D=1.04)$ with varying levels of anxiety and depression were recruited from a larger study examining personality, affective, and cognitive risk factors for psychopathology $(N=1123$; Warren et al., under review; analyses reported here are novel and are orthogonal to Warren et al., under review). From this larger study, participants were selected to be at risk for psychopathology according to their scores on dimensional measures of anxiety and depression (see Psychopathology questionnaires section under Measures). Specifically, participants were selected if they (1) scored at or above the 80th percentile on one of the three psychopathology dimensions and at or below the 50th percentile on the other two dimensions, (2) or if they scored at or above the 80th percentile on all three psychopathology dimensions, or (3) if they scored at or below the 50th percentile on all three psychopathology dimensions. All participants were right-handed, native speakers of English with self-reported normal color vision and hearing, with no neurological disorders or impairments. The Structured Clinical Interview for Axis I Disorders, NonPatient Edition (First et al., 1997) was administered to all participants. Although participants were not specifically selected based on DSM-IV-TR anxiety or mood disorder diagnosis, approximately $22 \%$ met criteria for anxiety disorder only (Anxiety NOS, Generalized Anxiety Disorder, Obsessive Compulsive Disorder, Post Traumatic Stress Disorder, Social Phobia), 9\% met criteria for mood disorder only (Major Depressive Disorder or Dysthymia), and 18\% met criteria for an anxiety and mood disorder. Participants were given a laboratory tour, informed of the procedures of the study, and screened for claustrophobia and other contraindications for MRI participation. The study was approved by the University of Illinois at Urbana-Champaign Institutional Review Board. Participants were excluded if they had ever experienced loss of consciousness $\geq 10$ min or exhibited current substance abuse or dependence, mania, or psychosis. Additional exclusion criteria included excessive motion or scanner artifact $(n=8)$, signal loss due to substantial uncorrected magnetic susceptibility in areas of interest $(n=1)$, or Stroop reaction time errors greater than 3 standard deviations from the sample mean $(n=1)$.

\section{MEASURES}

\section{Inhibition in everyday life}

The Behavior Rating Inventory of Executive Function (BRIEF; self-report version; Guy et al., 2004) is an ecologically sensitive, self-report questionnaire that measures several aspects of EF in an individual's everyday life, including inhibition. Through a series of item-level factor analyses using the BRIEF Warren et al. (under review), identified inhibition, shifting, and updating latent factors consistent with Miyake et al.'s (2000) EF framework. For the present study, the inhibition-item weights ( $\lambda s ; N=1123$ ) identified in Warren et al. (under review) were used to compute participants' behavioral inhibition in everyday life scores. The BRIEF inhibition factor score indexes an individual's ability to resist impulsive responses by pre-empting or stopping one's behavior at the appropriate time and the tendency to act prematurely without foresight in social contexts (Guy et al., 2004). Elevated scores represent impaired cognitive control, manifesting behaviorally as disinhibition and impulsivity. 


\section{Inhibition in the laboratory}

The color-word Stroop task was used as a measure of prepotent response inhibition. Participants completed the color-word Stroop task (Stroop, 1935) during fMRI data acquisition (see below) in which they were asked to press a button indicating the color of the ink in which color words and neutral words were printed, ignoring the dominant tendency to read the words. During the incongruent condition of the Stroop task, cognitive interference is created by the actual meaning of the presented word relative to the ink color in which it is presented (e.g., "RED" in blue ink).

Average RT for correct-response trials was computed for incongruent (e.g., "RED" in blue ink) and neutral trials (e.g., "LOT" in red ink). RT interference scores were computed by subtracting each participant's average neutral RT from their average incongruent RT, divided by their sum ([incongruent RT minus neutral RT]/[incongruent RT plus neutral RT]), and converted to $z$ scores across all subjects. Higher interference scores indicated that participants took longer to respond to the ink color of incongruent than of neutral words. No-response trials were excluded from behavioral analyses.

\section{Psychopathology questionnaires}

Dimensional measures of anxiety and depression, the Penn State Worry Questionnaire (PSWQ; Molina and Borkovec, 1994) and the Anxious Arousal and Anhedonic Depression scales of the Mood and Anxiety Symptom Questionnaire (MASQ; Watson et al., 1995b), were administered during the participant's first visit to the laboratory (see Table 1). Anxious apprehension was measured using the 16-item PSWQ (e.g., "My worries overwhelm me"). Anxious arousal was measured using the relevant 17-item subscale of the MASQ (MASQAA; e.g., "startled easily"). Anhedonic depression was measured using an 8-item subscale from the MASQ (MASQAD8; e.g., "Felt like nothing was very enjoyable"), as it has been shown to predict current and lifetime depressive disorders (Bredemeier et al., 2010). Past research has shown that these measures have good test-retest reliability and good convergent and discriminant validity in undergraduate and clinical samples (Watson et al., 1995a,b; Nitschke et al., 2001).

\section{Table 1 | Descriptive statistics.}

\begin{tabular}{lcccc}
\hline & M & SD & Min & Max \\
\hline QUESTIONNAIRE & & & & \\
1. PSWQ (Anxious apprehension) & 49.08 & 18.03 & 17 & 80 \\
2. MASQAA (Anxious arousal) & 27.56 & 7.58 & 17 & 48 \\
3. MASQAD8 (Anhedonic depression) & 16.89 & 5.77 & 8 & 33 \\
INHIBITION MEASURE & & & & \\
1. BRIEF factor score & 9.18 & 2.09 & 6.32 & 15.82 \\
2. RT interference & 0.11 & 0.60 & -0.30 & 0.23
\end{tabular}

$N=$ 85. PSWQ, Penn State Worry Questionnaire. MASQAA, Mood and Anxiety Symptom Questionnaire Anxious Arousal scale. MASQAD8, Mood and Anxiety Symptom Questionnaire Anhedonic Depression 8-item subscale. RT Interference computed by ([incongruent RT minus neutral RT]/[incongruent RT plus neutral RT]).

\section{EXPERIMENTAL TASK AND STIMULI Color-word Stroop task}

Participants completed color-word and emotion-word Stroop tasks during an fMRI session, and also completed an EEG procedure and a diagnostic interview in other sessions. Only findings from the color-word Stroop task during fMRI are presented here. Hemodynamic data from this same task for an overlapping set of participants was used in a separate study addressing an entirely different research question (Spielberg et al., 2011). The order of presentation of the two tasks within the fMRI session was counterbalanced. The color-word Stroop task consisted of blocks of color-congruent or color-incongruent words alternating with blocks of neutral words. Half of the trials in the congruent and incongruent blocks were neutral to prevent the development of word-reading strategies. This type of blocked-design color-word Stroop task has been shown to effectively elicit Stroop interference (Banich et al., 2000; Milham and Banich, 2005). There were eight orders of stimulus presentation blocks that were counterbalanced across subjects (each participant received one out of eight possible orders). In addition to the word blocks, there were four fixation blocks (one at the beginning, one at the end, and two in the middle of the session) and five rest blocks (one at the beginning, one at the end, and one between each word block). In the fixation condition, a fixation cross intensified in place of word presentation, and in the rest condition the subject was instructed to rest and keep their eyes open while the screen was blank.

Each trial consisted of one word presented in one of four ink colors (red, yellow, green, blue) on a black background, with each color occurring equally often with each word type. The task consisted of congruent trials in which the word named the ink color in which it was printed (e.g., the word "RED" printed in red ink), incongruent trials in which the word named a color incongruent with the ink color in which it was printed (e.g., "GREEN" printed in red ink), and neutral trials in which the word was unrelated to color (e.g., "LOT" in red ink). Neutral words were matched with color words on word frequency and length. Participants responded to the color of the ink with their middle and index fingers using left- and right-hand response boxes.

Participants received 256 trials presented in 16 blocks (four congruent, four incongruent, and eight neutral) of 16 trials each, with a variable ITI $( \pm 225 \mathrm{~ms})$ averaging $2000 \mathrm{~ms}$ between trial onsets. A trial began with the presentation of a word for $1500 \mathrm{~ms}$, followed by a fixation cross for an average of $500 \mathrm{~ms}$. There was a brief rest period after every fourth block. Additionally, there were four fixation blocks (one at the beginning, one at the end, and two in the middle) in which a brighter fixation cross was presented for $1500 \mathrm{~ms}$. Participants completed 32 practice trials during a low-resolution anatomical scan. No participants failed to understand the task instructions or the mapping between colors and buttons after completing practice trials. Stimuli, word presentation, and reaction-time measurement were controlled by STIM software (James Long Company, Caroga Lake, NY).

\section{Image acquisition}

A series of 370 fMRI images (16 images per block of 16 stimuli plus rest and fixation periods) were acquired using a gradientecho echo-planar pulse sequence (TR $2000 \mathrm{~ms}$, TE $25 \mathrm{~ms}$, flip 
angle $80^{\circ}, \mathrm{FOV}=22 \mathrm{~cm}$ ) on a $3 \mathrm{~T}$ Siemens Allegra head-only scanner. Thirty-eight contiguous oblique axial slices (slice thickness $3 \mathrm{~mm}$, in-plane resolution $3.4375 \times 3.4375 \mathrm{~mm}^{2}, 0.3 \mathrm{~mm}$ gap between slices) were acquired parallel to the anterior and posterior commissures. After the EPI sequence, a 160-slice MPRAGE structural sequence was acquired (slice thickness $1 \mathrm{~mm}$, in-plane resolution $1 \times 1 \mathrm{~mm}$ ) for registering each participant's functional data to standard space. Prior to the EPI sequence, standard Siemens magnetic field maps were collected with the same slice prescription as the functional scans using a multi-echo gradient echo acquisition (TE's of 10 and $12.46 \mathrm{~ms}$ ). This field map was used for correction of geometric distortions in the EPI data caused by magnetic field inhomogeneity.

\section{fMRI data reduction and analysis}

Functional image processing and analysis relied on tools from the FSL analysis package (e.g., MCFLIRT, PRELUDE, FILM, FUGUE, FEAT, FLAME; http://www.fmrib.ox.ac.uk/fsl) and AFNI (http:// afni.nimh.nih.gov/afni/). Additional region-of-interest (ROI) analyses were carried out using locally written Matlab programs (e.g., Herrington et al., 2005) and IBM SPSS Statistics version 19.0 .

Functional data for each participant were motion-corrected using rigid-body registration, implemented in FSL's linear registration tool, MCFLIRT (Jenkinson et al., 2002). Temporal lowpass filtering was carried out using AFNI's 3dDespike tool (http:// afni.nimh.nih.gov/) to remove intensity spikes. The ends of two participants' time series were truncated due to excessive motion. All other participants demonstrated less than $3.3 \mathrm{~mm}$ absolute motion or $2 \mathrm{~mm}$ relative motion. After motion correction and temporal low-pass filtering, each time series was corrected for geometric distortions caused by magnetic field inhomogeneity (Jezzard and Balaban, 1995; Jenkinson, 2004). Remaining preprocessing steps, single-subject statistics, and group statistics were completed with FEAT. The first three volumes of each participant's functional data were discarded to allow the MR signal to reach a steady state. Each time series was temporally filtered with a nonlinear high-pass filter to attenuate frequencies below $1 / 212 \mathrm{~Hz}$ (to remove drift in signal intensity), mean-based intensity-normalized by the same single scaling factor, and spatially smoothed using a 3D Gaussian kernel (FWHM $5 \mathrm{~mm}$ ) prior to analysis.

Blood-oxygen-level-dependent (BOLD) activity during the color-word Stroop task was assessed using FILM (FMRIB's Improved Linear Model). Statistical maps were generated via multiple regression on each intracerebral voxel (Woolrich et al., 2001). An explanatory variable (EV) was created for each trial type (congruent, neutral, incongruent, and rest; fixation condition left unmodeled) and convolved with a gamma function to better approximate the temporal course of the BOLD hemodynamic response function (e.g., Aguirre et al., 1998). The contrast of particular interest for this study is the incongruent versus neutral contrast, because incongruent trial performance requires executive function to exert top-down control and resolve conflict. Each EV (i.e., regressor) yielded a per-voxel effect-size parameter estimate (ß) map representing the magnitude of activity associated with that EV for a given participant. Functional activation maps for each contrast were transformed into MNI stereotactic space (ICBM152 2009a Nonlinear Symmetric, $1 \times 1 \times 1 \mathrm{~mm}$ T1 Atlas; Fonov et al., 2009) using FMRIB's Non-Linear Image Registration Tool, FNIRT (Andersson et al., 2007).

Group inferential statistical analyses were carried out using FLAME and SPSS. To identify ROIs for subsequent analysis, activated voxels were identified for the incongruent vs. neutral contrast via two-tailed, per-voxel $t$-tests on contrast $\beta$ maps converted to $\mathrm{z}$-scores. Monte Carlo simulations via AFNI's AlphaSim program estimated the overall significance level (probability of a false detection) for thresholding these 3D functional $\mathrm{z}$-map images (Ward, 2000). These simulations used a gray-matter mask to limit the number of voxels under consideration $\left(2340 \mathrm{~mm}^{3}\right)$ and provided a cluster size (390) and $z$-value $(z=2.97$; corresponding $p$-value $=0.003$ ) combination to use for thresholding, resulting in an overall family-wise error rate of 0.05 . In order to explore brain regions uniquely associated with inhibition-related constructs, BRIEF inhibition factor score and RT interference (each converted to $z$ scores) for each participant were entered as predictors in whole-brain, per voxel, cross-subject regression analyses in FSL. Updating and shifting factor scores (Warren et al., under review) were entered as covariates in order to isolate the specific effects of inhibition. Although there is empirical support for moderate correlations and some overlap among some aspects of EF (Warren et al., under review), inhibition, updating, and shifting components are also behaviorally, genetically, and neurally dissociable (e.g., Miyake et al., 2000; Collette et al., 2005; Friedman et al., 2008; Warren et al., under review). Thus, in order to isolate the specific effects of inhibition in everyday life and the type of inhibition typically observed in the laboratory, brain activity showing distinct relationships with BRIEF inhibition and RT interference was examined by including all EF measures (BRIEF inhibition factor score, RT interference, updating and shifting factor scores) simultaneously in one regression model. This regression analysis produced a $\beta$ map corresponding to the unique variance associated with each inhibition construct.

Clusters associated with inhibition in everyday life and RT interference that surpassed statistical thresholding were identified as ROIs. To assess the potential effect of psychopathology on neural activity related to these specific inhibition processes, a score for each ROI identified in which BRIEF inhibition factor score and RT interference predicted fMRI was created by averaging $\beta$ values across voxels in each ROI, for each participant. ROI scores were then entered as the dependent variable in three separate hierarchical linear regressions: (1) PSWQ, MASQAA, and MASQAD8 were entered together as independent variables, (2) their two-way interactions were added together, and (3) their three-way interaction was added. In order to illustrate the resulting moderating effects of psychopathology on ROIs, interactions were plotted and simple slopes tested whether the relationship between brain activity and psychopathology was significantly different from zero at different combinations of high and low levels of anxiety types (see Engels et al., 2010, for details of a similar approach). In Figures 2-4, the relationship between brain activity and anxious apprehension was plotted at high and low levels of anxious arousal. In Figure 5, the relationship between brain activity and depression was plotted at high and low levels of anxious 
apprehension. For all figures that plot interactions, "high" and "low" refer to $\pm 1 S D$.

\section{RESULTS}

\section{BEHAVIORAL DATA}

All participants demonstrated color-choice accuracy of at least $85 \%$. As a manipulation check, we examined RT interference for color-word trials. As expected, participants demonstrated more RT interference for incongruent-word trials $(M=814 \mathrm{~ms}, S D=$ $160 \mathrm{~ms})$ than for congruent-word trials $(M=633 \mathrm{~ms}, S D=$ $103 \mathrm{~ms}), t_{(84)}=15.3, p<0.001$, and neutral-word trials $(M=$ $652 \mathrm{~ms}, S D=103 \mathrm{~ms}), t_{(84)}=15.2, p<0.001$.

Descriptive statistics for all of the measures are presented in Table 1, and zero-order correlations among psychopathology and inhibition measures are presented in Table $2^{1}$.

\section{fMRI DATA}

\section{Brain regions uniquely associated with BRIEF inhibition}

Table 3 lists seven regions that were positively correlated with the BRIEF inhibition factor score. In line with hypotheses, higher BRIEF inhibition factor scores were associated with more activation in left mid-DLPFC (middle frontal gyrus; see Figure 1) and left IFG, regions that are generally associated with implementing inhibition-related processes. Additional clusters emerged in frontal pole, OFC, and supramarginal and angular gyrus regions. There were no significant clusters negatively correlated with BRIEF inhibition factor scores.

\section{Moderation of brain activity by psychopathology associated with BRIEF inhibition factor score}

No significant moderation by anxiety, depression, or their interactions emerged with any of the self-reported inhibition ROIs.

\section{Brain regions uniquely associated with $\mathrm{RT}$ interference}

Table 4 lists a network of regions that were negatively correlated with RT interference. In line with hypotheses, higher RT interference was associated with less activation in left posterior DLPFC (middle frontal gyrus), bilateral IFG, and ACC (rostral, dorsal,

\section{Table 2 | Zero-order correlations among psychopathology and} inhibition-related measures.

\begin{tabular}{lllll}
\hline Measure & $\mathbf{1}$ & $\mathbf{2}$ & $\mathbf{3}$ & $\mathbf{4}$ \\
\hline 1. PSWQ (Anxious apprehension) & - & & & \\
2. MASQAA (Anxious arousal) & $0.48^{* *}$ & - & & \\
3. MASOAD8 (Anhedonic depression) & $0.49^{* *}$ & $0.51^{* *}$ & - & \\
4. BRIEF inhibition factor score & 0.10 & $0.35^{* *}$ & $0.29 * *$ & - \\
5. RT interference & 0.12 & 0.13 & 0.11 & 0.13 \\
\hline
\end{tabular}

${ }^{*} p \leq 0.01$ (two-tailed).

\footnotetext{
${ }^{1}$ Given the significant zero-order correlations among the BRIEF Inhibition Factor Score and psychopathology questionnaires, there is a potential for selection bias with respect to brain activity in regions of interest. However, given that the psychopathology measures did not significantly moderate the relationship between brain activity and BRIEF Factor Score, the potential for non-independence is not an issue.
}

and anterior mid-cingulate), as well as regions that are generally associated with attentional control and motor response coordination (e.g., premotor cortex, frontal eye fields, posterior parietal cortex, precuneus; see Figure 1). Additional clusters emerged in occipital cortex, thalamus and caudate, parahippocampal gyrus, frontal pole, OFC, and supramarginal and angular gyrus regions (see Figure 1). There were no significant clusters positively correlated with RT interference.

\section{Moderation of brain activity by psychopathology associated with RT interference}

No significant main effect of anxiety type, depression, or their three-way interaction emerged. Table 5 lists regions with twoway interactive effects for anxiety and depression for responseinhibition-related brain activity. Three regions were moderated by four, two-way interactions. A PSWQ $\times$ MASQAA interaction emerged for left posterior DLPFC (Figure 2). As illustrated in Figure 2, increased anxious apprehension was associated with increased left posterior DLPFC activation, but only when anxious arousal was low. Tests of simple slopes showed that this was the only significant slope $\left[t_{(78)}=2.84, p<0.01\right]$. A PSWQ $\times$ MASQAA interaction was found for right middle temporal gyrus (MTG; Figure 3). Tests of simple slopes showed that increased anxious apprehension was associated with decreased right MTG activation at high levels of anxious arousal $\left[t_{(78)}=\right.$ $-2.86, p<0.01$ ] but with increased activation at low levels of anxious arousal $\left[t_{(78)}=2.02, p=0.05\right]$. Finally, two interactions emerged for right frontal pole (Figures 4 and 5). Similar to right MTG, increased anxious apprehension was associated with decreased right frontal pole activation at high levels of anxious arousal $\left[t_{(78)}=-3.47, p<0.001\right]$ but with increased activation at low levels of anxious arousal $\left[t_{(78)}=2.91, p<0.01\right.$; Figure 4]. Additionally, a PSWQ $\times$ MASQAD8 interaction emerged in which high levels of anhedonic depression were associated with decreased right frontal pole activity at low levels of anxious apprehension. Tests of simple slopes showed that this was the only significant slope $\left[t_{(78)}=-3.55, p<0.001\right.$; Figure 5].

\section{DISCUSSION}

The present study examined neural mechanisms supporting ecologically sensitive versus laboratory-based measures of inhibitory function in order to clarify the broader construct of inhibition as well as their role in psychopathology. Brain-activation results were consistent with regions of interest predicted to be associated with inhibition-related processes. In general, worse self-reported inhibition in everyday life (elevated BRIEF factor score) was associated with increased activity in brain regions typically associated with inhibitory function (left DLPFC, left IFG, bilateral inferior parietal cortex; Figure 1). In contrast, worse performance on the laboratory task (increased RT interference) was associated with decreased brain activity in these regions as well as ACC (see Figure 1). Importantly, although DLPFC activity was associated with both measures of inhibitory functions, each measure exhibited unique relationships with DLPFC. As predicted, worse self-reported inhibition was associated with increased activity in mid-DLPFC, and greater RT interference was associated with less activity in posterior DLPFC. These differential patterns 
Table 3 | Distinct effects of brief inhibition factor score.

\begin{tabular}{|c|c|c|c|c|c|c|c|c|}
\hline Region & Cluster size & Mean Z & \multicolumn{3}{|c|}{ COM Location } & \multicolumn{3}{|c|}{ Max Z Location } \\
\hline L frontal pole, OFC & 397 & 3.30 & -46 & 39 & -16 & -48 & 40 & -17 \\
\hline L inferior frontal gyrus (IFG), anterior insula & 1346 & 3.25 & -46 & 16 & 0 & -51 & 17 & -2 \\
\hline L frontal pole, IFG-pars triangularis & 423 & 3.35 & -47 & 39 & 6 & -46 & 40 & 6 \\
\hline L supramarginal gyrus & 4851 & 3.26 & -54 & -53 & 41 & -54 & -44 & 52 \\
\hline $\mathrm{R}$ angular gyrus, lateral occipital cortex & 558 & 3.31 & 48 & -55 & 54 & 50 & -56 & 54 \\
\hline
\end{tabular}

$N=85 . C O M$, center of mass; $R$, right; L, left; DLPFC, dorsolateral prefrontal cortex; OFC, orbitofrontal cortex; TPJ, temporoparietal junction.

${ }^{a} z$-scores $>2.9677$, cluster-size $\geq 390$ (corrected $p<0.05$ ).

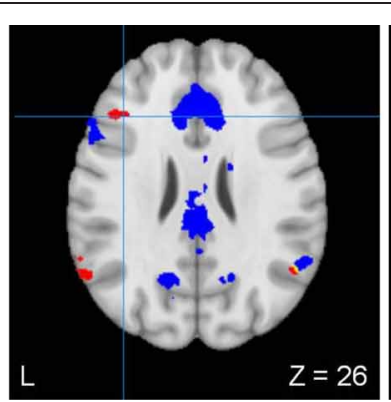

FIGURE 1 | Areas of activation uniquely associated with either self-reported inhibition in everyday life or prepotent response inhibition. Red, increased brain activation associated with behavioral inhibition as measured by BRIEF inhibition factor score. Blue, decreased brain activation
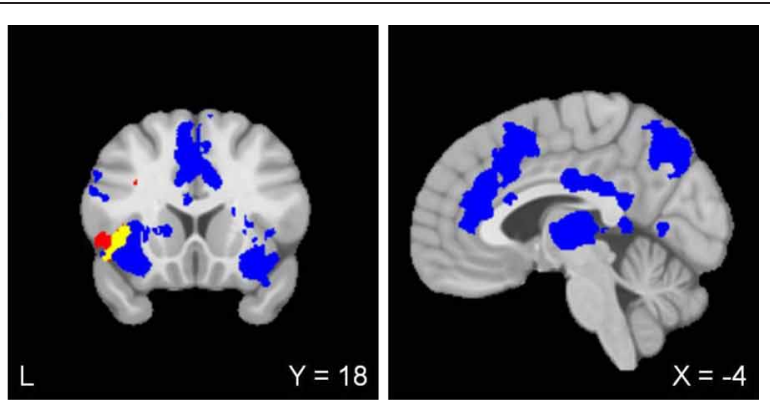

associated with prepotent response inhibition as measured by RT interference. Yellow, brain activation overlap between BRIEF inhibition factor score and RT interference. L, Left. Location of crosshairs emphasizes a differentiation of mid-DLPFC (red) and posterior DLPFC (blue) regions. of inhibition-related processes suggest a distinct role for each DLPFC area.

The cascade of control model (Banich et al., 2000, 2009; Milham and Banich, 2005; Banich, 2009) identifies aspects of EF that are critical for inhibiting responses, including biasing responses toward task-relevant processes, biasing attention toward task-relevant representations, response selection, and response evaluation. This model proposes that distinct areas of DLPFC implement these functions, which are necessary for cognitive control. Posterior DLPFC imposes a top-down attentional set toward task-relevant processes, maintains the overall task goals, and subsequently biases other brain regions (e.g., mid-DLPFC, dorsal ACC, parietal cortex) toward processing taskrelevant information. In contrast, mid-DLPFC is involved in selecting and maintaining the most relevant aspects of task stimuli (Banich, 2009) and has been suggested to play an important role in stimulus-driven attentional control (Crocker et al., 2012). MidDLPFC is thought to be involved in interrupting top-down processing to reorient attention to stimuli that have been identified as relevant (Corbetta et al., 2008; Crocker et al., 2012) and therefore could be said to be critically involved in tracking and multitasking functions. In the context of present findings, a behavioral manifestation of a high BRIEF inhibition factor score is impulsivity. Thus, mid-DLPFC hyperactivity associated with increased
BRIEF inhibition factor score could reflect paying attention to too many task representations and/or hyper-focusing on stimulus properties, which could disrupt relevant task goal maintenance. In line with this interpretation, hyperactivity in mid-DLPFC has been linked to over-engagement with irrelevant features of stimuli (the meaning of threat-related words in an emotion-word Stroop task), interfering with processing task-relevant features (word color; Engels et al., 2010).

In contrast, a negative correlation between RT interference and posterior DLPFC was observed, such that the greater the RT interference, the less the brain activity. Given DLPFC's prominent role in top-down attentional control (Milham et al., 2003), if posterior DLPFC fails to impose a top-down attentional set toward task-relevant processes (inferred by decreased activity), one would anticipate greater RT interference. Present results are consistent with other findings (Banich et al., 2000; Milham et al., 2003; Milham and Banich, 2005).

In line with the cascade-of-control model, RT interference was also associated with areas of ACC that are involved in response selection and response evaluation. Specifically, the model asserts that there is a temporal cascade of cognitive operations, such that, following DLPFC activation, dorsal ACC selects the appropriate response among available response options. When incorrect responses are made during a task, more anterior regions of ACC 
Table 4 | Distinct effects of RT interference.

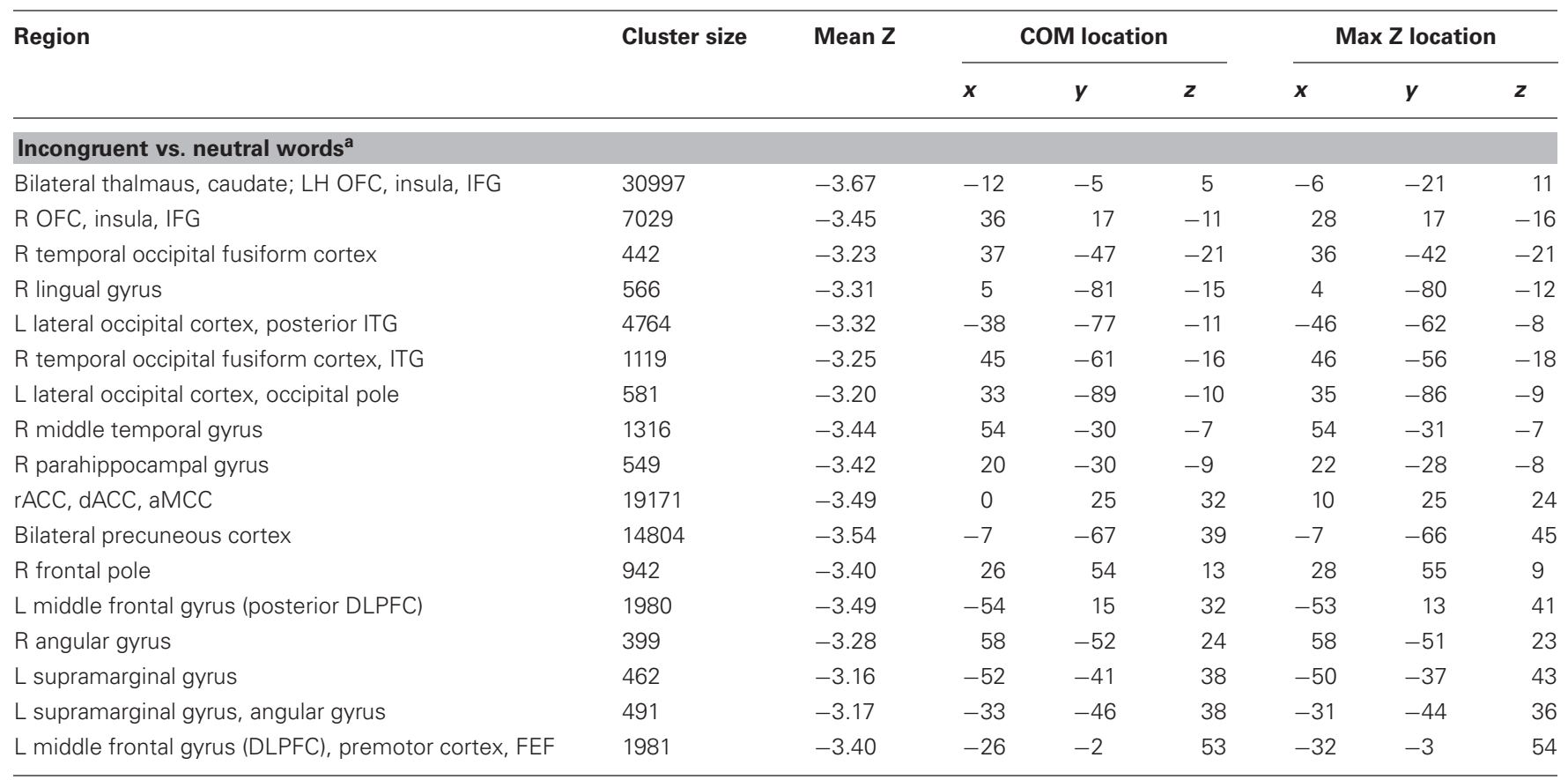

$N=85 . C O M$, center of mass; $R$, right; $L$, left; DLPFC, dorsolateral prefrontal cortex; OFC, orbitofrontal cortex; IFG, inferior frontal gyrus; ITG, inferior temporal gyrus; $A A C C$, dorsal anterior cingulate cortex; rACC, rostral anterior cingulate cortex; aMCC, anterior mid-cingulate cortex; FEF, frontal eye field.

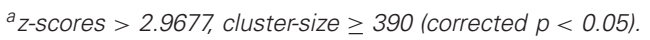

Table 5 | Regression analyses for two-way interactive effects of anxiety and depression on RT interference ROIs.

\begin{tabular}{|c|c|c|c|c|c|}
\hline Region & & $R^{2}$ & $\Delta R^{2}$ & Test & $p$ \\
\hline \multirow[t]{2}{*}{ L middle frontal gyrus (posterior DLPFC) } & PSWO $\times$ MASQAA & & 0.08 & $t_{(78)}=-2.65$ & 0.01 \\
\hline & Full model & 0.156 & & $F_{(6,78)}=2.40$ & 0.04 \\
\hline R middle temporal gyrus & Full model & 0.164 & & $F_{(6,78)}=2.55$ & 0.03 \\
\hline R frontal pole & PSWO $\times$ MASQAA & & 0.13 & $t_{(78)}=-3.48$ & $<0.01$ \\
\hline R frontal pole & Full Model & 0.185 & & $F_{(6,78)}=3.00$ & 0.01 \\
\hline
\end{tabular}

N, 85. PSWQ, Penn State Worry Questionnaire; MASQAA, Mood and Anxiety Symptom Questionnaire Anxious Arousal scale; MASQAD8, Mood and Anxiety Symptom Questionnaire Anhedonic Depression 8-item subscale; $R$, right; $L$, left; DLPFC, dorsolateral prefrontal cortex. $\Delta R^{2}$ is the incremental variance associated with the interaction term, with its individual constituents already in the model.

signal posterior DLPFC to assert greater top-down control for task performance, requiring re-initiation of certain steps in the temporal cascade of events. In addition to posterior DLPFC and ACC, present findings for regions of activation for RT interference were consistent with those implicated in a distributed network associated with response inhibition, including bilateral IFG, as well as regions that are generally associated with attentional control and coordinating motor responses (e.g., premotor cortex, frontal eye fields, posterior parietal cortex, precuneus; Corbetta et al., 2008; Banich, 2009).

Contributing to understanding EF deficits in psychopathology, select patterns of brain activation for response inhibition (RT interference) were modulated by anxiety and depression.
A two-way interaction emerged for left posterior DLPFC in which greater activity was associated with high anxious apprehension, but only when anxious arousal was low. Anxious apprehension typically involves elaborated verbal processing and worry. Given that posterior DLPFC is involved in imposing top-down attentional control and maintaining task set, greater activity in this area may reflect an attempt to compensate for anxious apprehension (which can be inferred to impair the efficiency of this inhibition-related function). Considerable evidence suggests that anxiety is often associated with increased susceptibility to distraction (see Derakshan and Eysenck, 2009, for review), hypothesized to reflect impaired inhibition (e.g., Eysenck and Derakshan, 2011). According to attentional control theory, anxiety impairs 

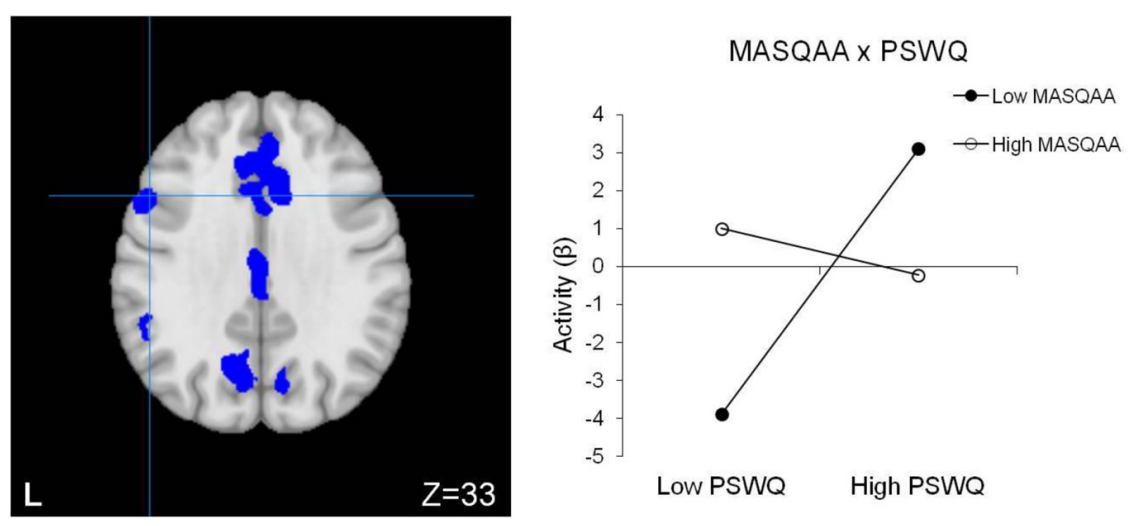

FIGURE 2 | Posterior DLPFC activation for RT interference. Blue, decreased brain activation associated with RT interference. L, left. Graphing the MASQAA $\times$ PSWQ interaction illustrates that anxious apprehension's relationship with left posterior DLPFC depends on the level of co-occurring anxious arousal.
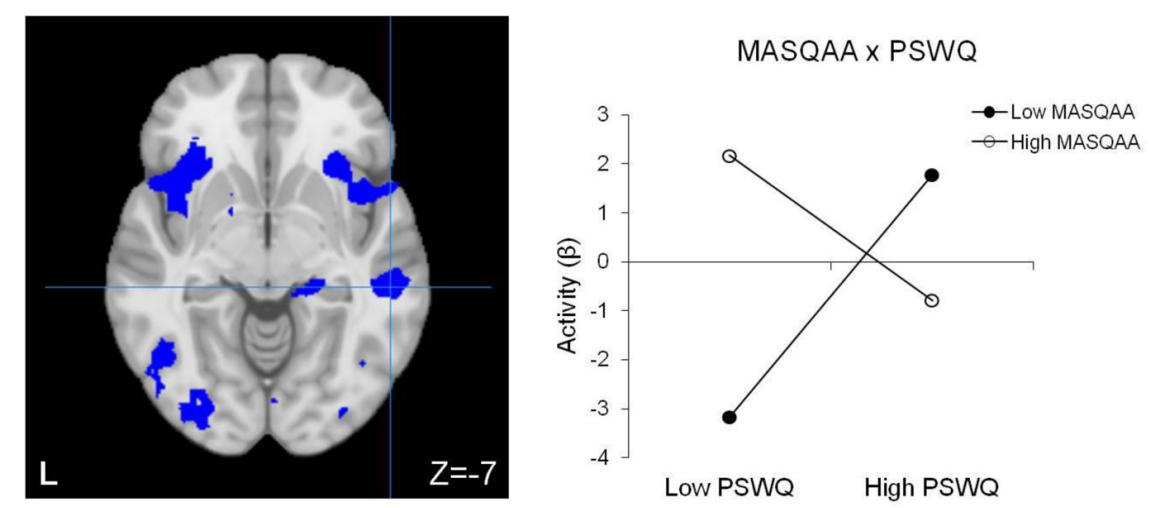

FIGURE 3 | Right MTG activation for RT interference. Blue, decreased brain activation associated with RT interference. L, left. Graphing the MASQAA $\times$ PSWQ interaction illustrates that anxious apprehension's relationship with right MTG depends on the level of co-occurring anxious arousal.
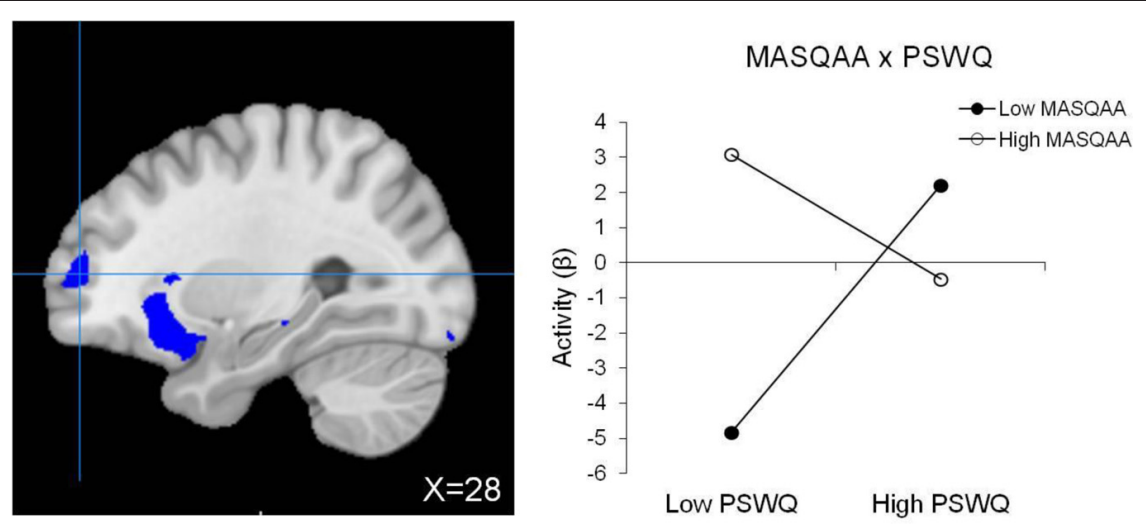

FIGURE 4 | Right frontal pole activation for RT interference. Blue, decreased brain activation associated with RT interference. Graphing the MASOAA $\times$ PSWQ interaction illustrates that anxious apprehension's relationship with right frontal pole depends on the level of co-occurring anxious arousal.

processing efficiency to a greater extent than it impairs performance effectiveness (i.e., quality of performance; Eysenck et al., 2007) and manifests in greater activation in brain regions associated with attentional control. Present findings suggest that individuals high in anxious apprehension (worry), a specific dimension of anxiety, especially when anxious arousal is low, are more susceptible to distraction and thus to impaired efficiency of inhibition during cognitively demanding tasks (i.e., inhibiting the 

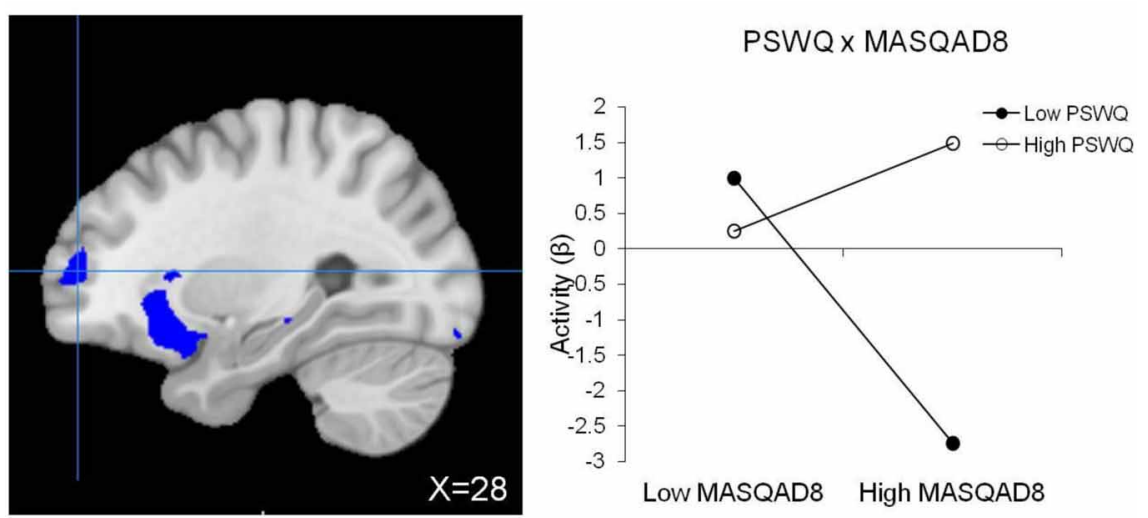

FIGURE 5 |Right frontal pole activation for RT interference (same region pictured in Figure 4). Blue, decreased brain activation associated with RT interference. Graphing the PSWQ $\times$ MASQAD8 interaction illustrates that anxious apprehension's relationship with right frontal pole depends on the level of co-occurring anhedonic depression. dominant tendency to read the color word). The fact that anxious apprehension and anxious arousal are not associated with error rate could reflect compensation by posterior DLPFC (inferred from greater activity). Despite its disrupting impact on efficiency, increased activity associated with anxious apprehension or worry may be adaptive under some circumstances. Anxious apprehension ameliorated a depression-related suppression of activity in DLPFC (again, only when anxious arousal was low; Engels et al., 2010). Types of anxiety and depression may thus interact to influence optimal levels of activity in brain regions associated with cognitive control, which in turn may affect the balance of goal maintenance vs. stimulus-driven or contextual processing.

A two-way interaction emerged for right MTG in which greater RT interference activity was associated with high anxious apprehension when anxious arousal was low and with decreased activity when anxious arousal was high. Additional examination of this interaction revealed one significant slope, such that brain activity increased as anxious arousal increased, but only when anxious apprehension was low. Right MTG is thought to interact with a network of regions involved in detecting and responding to threat (e.g., Compton et al., 2003; Corbetta et al., 2008). This region may be a part of a system that functions adaptively to switch between top-down attentional control and more stimulus-driven processing (Corbetta et al., 2008). Using an emotion-word Stroop task, Engels et al. (2007) demonstrated that negative emotion words elicited greater right middle-temporal/inferior-temporal activity in an anxious arousal group. Importantly, present results generalize Engels' et al. (2007, 2010) findings to non-emotional contexts, suggesting that anxiety-modulated increases in activity in this region interfere with an inhibition-related function for cognitive control. Additionally, in a non-overlapping sample, Engels et al. (2010) found that anxious arousal ameliorated depression-related suppression of activity in this region, in response to threatening words. Again, these findings suggest that under some circumstances anxiety-related activation has an adaptive function.

Similar to the pattern observed for right MTG, greater right frontal pole (BA10) activity was associated with high anxious apprehension when anxious arousal was low and with decreased activity when anxious arousal was high. Additionally, anxious apprehension diminished depression-related suppression of activity in this region. Rostral PFC (BA10) has been implicated in supporting a wide range of functions including prospective memory, multitasking, and "mentalizing" or reflecting on mental states (see Burgess et al., 2007, for review). According to the gateway hypothesis (Burgess et al., 2007), rostral PFC is part of a cognitive control system that biases the relative influence of stimulus-independent and stimulus-oriented thought. Lateral regions of rostral PFC are associated with stimulus-independent cognition, the mental processes that accompany self-generated or self-maintained thought that is not provoked by or directed toward an external stimulus (i.e., task-irrelevant thought). The right frontal pole region in the present study overlaps with the lateral area of rostral PFC identified by Burgess et al. (2007) as supporting stimulus-independent function. Anxious apprehension modulation of brain activity in this region (when anxious arousal is low) could reflect task-irrelevant thoughts such as worry, an example of stimulus-independent cognition, potentially interfering with task efficiency. However, anxious apprehension also interacted with depression in this same region, such that depression-related hypoactivity decreased as anxious apprehension increased. Findings suggest that whereas anxious apprehension could interfere with task efficiency when anxious arousal is low, worry could potentially be adaptive for task performance at high levels of depression.

Contrary to hypotheses, no significant moderation of anxiety, depression, or their interactions emerged with any of the ROIs associated with self-reported inhibition in everyday life. It is possible that the color-word Stroop task does not robustly engage inhibition-related neural mechanisms that implement the kind of everyday inhibitory control that is affected by anxiety or depression. Another possible explanation for the lack of significant findings is the general nature and range of everyday scenarios that the self-reported inhibition score indexes. Although the selfreported inhibition score may be sensitive to neural mechanisms supporting this function, the measure may not be specific enough to capture anxiety and depression deficits. 
Maintenance of top-down attentional control is typically assumed to be the main function of DLPFC. However, present results suggest a more nuanced view of DLPFC in the context of cognitive control, as sub-regions were differentiated by two aspects of inhibition-related functions. Present results support an emerging view that areas within DLPFC (mid and posterior) may provide distinct contributions to cognitive control (Banich, 2009). Whereas mid-DLPFC has been associated with stimulus-driven attentional control (Crocker et al., 2012), posterior DLPFC imposes a top-down attentional set that maintains overall task goals. In combination, these regions are involved in preventing irrelevant information from entering working memory. In the context of the current study, present findings suggest that differing inhibition-related mechanisms may contribute to the efficiency in which information is maintained in working memory, as well as resistance to interference.

DLPFC dysfunction has been implicated as a source of cognitive impairment in a range of psychopathology, including depression and anxiety (Engels et al., 2007, 2010; Levin et al., 2007; Warren et al., 2008; Herrington et al., 2010; Silton et al., 2010). Although inhibitory functions are not the only factors that are associated with cognitive dysfunction in psychopathology, their differing neural mechanisms certainly have probative value. For example, theories of depression (Joormann et al., 2007) and anxiety (Eysenck et al., 2007) postulate inhibitory dysfunction as a source of symptom development and maintenance, although specific inhibitory functions are not addressed. Indeed, present findings demonstrate that only response inhibitionrelated brain activity (RT interference) was significantly moderated by psychopathology. Thus, assessing individual differences in specific inhibition-related functions and their neural mechanisms might be a profitable approach to understanding how "inhibition" contributes to cognitive and emotional disruptions in psychopathology.

\section{REFERENCES}

Aguirre, G. K., Zarahn, E., and D’Esposito, M. (1998). The variability of human, BOLD hemodynamic responses. Neuroimage 8, 360-369. doi: 10.1006/nimg. 1998. 0369

American Psychiatric Association. (2000). Diagnostic and Statistical Manual of Mental Disorders (4thed., text rev.). Washington, DC: Author.

Aron, A. R., Robbins, T. W., and Poldrack, R. A. (2004). Inhibition and the right inferior frontal cortex. Trends Cogn. Sci. 8, 170-177. doi: 10.1016/j.tics.2004.02.010

Andersson, J. L. R., Jenkinson, M., and Smith, S. (2007). Non-linear Optimization (Tech. Rep. TR07JA1). Oxford, UK: University of Oxford, FMRIB Centre.

Anticevic, A., Cole, M. W., Murray, J. D., Corlett, P. R., Wang, X.J., and Krystal, J. H. (2012). The role of default network deactivation in cognition and disease.
Trends Cogn. Sci. 16, 584-592. doi: 10.1016/j.tics.2012.10.008

Andrews, V. H., and Borkovec, T. D. (1988). The differential effects of inductions of worry, somatic anxiety, and depression on emotional experience. J. Behav. Ther. Exp. Psychiatry 19, 21-26. doi: 10.1016/0005-7916(88)90006-7

Banich, M. T. (2009). Executive function: the search for an Psychol. Sci. 18, 89-94. doi: 10.1111/j.1467-8721.2009.01615.x

Banich, M. T., Mackiewicz, K. L., Depue, B. E., Whitmer, A., Miller, G. A., and Heller, W. (2009). Cognitive control mechanisms, emotion, \& memory: a neural perspective with implications for psychopathology. Neurosci. Biobehav. Rev. 33, 613-616. doi: 10.1016/j.neubiorev.2008.09.010

Banich, M. T., Milham, M. P., Atchley, R. A., Cohen, N. J., Webb, A., Wszalek, T., et al. (2000). Prefrontal integrated account. Curr. Dir.

Anxiety-modulated hyperactivity in brain regions associated with cognitive control suggests a vulnerability to distraction, even in conditions when there is no manipulated threat (e.g., colorword Stroop task). In the same vein, Silton et al. (2011) found that, as anxious apprehension increased, increased dACC activity (another key region associated with implementing cognitive control) was associated with greater Stroop interference (less efficient performance). Neuroimaging evidence and theories of anxiety (e.g., Eysenck et al., 2007; Eysenck and Derakshan, 2011) suggest that excessive anxiety manifests as hyperactivity in brain regions associated with attentional control during task performance, a pattern of activity that is thought to reflect compensation. However, there are limits to compensation, and it is important to determine when compensation may break down, such as when individuals with excessive anxiety are under stress. Under such conditions, it is likely that functional impairments become overtly apparent in the contexts in which they are most detrimental (e.g. during an exam or meeting an important deadline).

Present findings reveal specific inhibition-related neural mechanisms associated with PFC, particularly sub-regions of DLPFC, and MTG, as well as the modulating effects of specific combinations of anxious apprehension, anxious arousal, and anhedonic depression. Although these effects indicate potential sources of impaired or disrupted performance, under some circumstances they may function to ameliorate or compensate for imbalances in optimal levels of activity in systems of cognitive control.

\section{ACKNOWLEDGMENTS}

This research was supported by NIMH (P50 MH079485, R01 MH61358, T32 MH19554) and the University of Illinois Department of Psychology. We would like to thank Katherine Mimnaugh for assistance in participant recruitment and data collection.

regions play a predominant role in imposing an attentional 'set': evidence from fMRI. Cogn. Brain Res. 10, 1-9. doi: 10.1016/S09266410(00)00015-X

Barlow, D. H. (2002). Anxiety and Its Disorders: The Nature and Treatment of Anxiety and Panic, 2nd Edn. New York, NY: Guilford Press.

Barlow, D. H. (1991). Disorders of emotion. Psychol. Inquiry 2, 58-71. doi: 10.1207/s15327965pli0201_15

Basso, M. R., Lowery, N., Ghormley, C., Combs, D., Purdie, R., and Neel, J. (2007). Comorbid anxiety corresponds with neuropsychological dysfunction in unipolar depression. Cogn. Neuropsychiatry 12, 437-456. doi: 10.1080/135468 00701446517

Bredemeier, K., Spielberg, J. M., Silton, R. L., Berenbaum, H., Heller, W., and Miller, G. A. (2010). Screening for depressive disorders using the mood anxiety symptoms Questionnaire
Anhedonic Depression Scale: a receiver-operating characteristic analysis. Psychol. Assess. 22, 702-710. doi: 10.1037/a0019915

Burgess, P. W., Alderman, N., Volle, E., Benoit, R. G., and Gilbert, S. J. (2009). Mesulam's frontal lobe mystery re-examined. Restorat. Neurol. Neurosci. 27, 493-506. doi: 10.3233/RNN-2009-0511

Burgess, P. W., Gilbert, S. J., and Dumontheil, I. (2007). Function and localization within rostral prefrontal cortex (area 10). Philos. Trans. R. Soc. London B Biol. Sci. 362, 887-899. doi: 10.1098/rstb.2007.2095

Collette, F., Van der Linden, M., Laureys, S., Delfiore, G., Degueldre, C., Luxen, A., et al. (2005). Exploring the unity and diversity of the neural substrates of executive functioning. Hum. Brain Mapp. 25, 409-423. doi: 10.1002/hbm.20118

Compton, R. J., Banich, M. T., Mohanty, A., Milham, M. P., 
Herrington, J., Miller, G. A., et al. (2003). Paying attention to emotion: An fMRI investigation of cognitive and emotional Stroop tasks. Cogn. Affect. Behav. Neurosci. 3, 81-96. doi: 10.3758/ CABN.3.2.81

Corbetta, M., Patel, G., and Shulman, G. L. (2008). The reorienting system of the human brain: from environment to theory of mind. Neuron 58, 306-324. doi: 10.1016/j.neuron.2008.04.017

Crocker, L. D., Heller, W., Spielberg, J. M., Warren, S. L., Bredemeier, K., Sutton, B. P., et al. (2012). Neural mechanisms of attentional control differentiate trait and state negative affect. Front. Psychol. 3:289.doi: 10.3389/fpsyg.2012.00298

Derakshan, N., and Eysenck, M. W. (2009). Anxiety, processing efficiency, and cognitive performance: new developments from attentional control theory. Eur. Psychol. 14, 168-176. doi: 10.1027/1016-9040.14.2.168

Dosenbach, N. U. F., Fair, D. A., Cohen, A. L., Schlaggar, B. L., and Petersen, S. E. (2008). A dualnetworks architecture of top-down control. Trends Cogn. Sci. 12, 99-105. doi: 10.1016/j.tics.2008. 01.001

Emmanuel, J., Simmonds, S., and Tyrer, P. (1998). Systematic review of the outcome of anxiety and depressive disorders. Br. J. Psychiatry Suppl. 173, 35-41.

Engels, A. S., Heller, W., Mohanty, A., Herrington, J. D., Banich, M. T., Webb, A. G., et al. (2007). Specificity of regional brain activity in anxiety types during emotion processing. Psychophysiology 44, 352-363. doi: 10.1111/j.1469-8986. 2007.00518.x

Engels, A. S., Heller, W., Spielberg, J. M., Warren, S. L., Sutton, B. P., Banich, M. T., and Miller, G. A. (2010). Co-occurring anxiety influences patterns of brain activity in depression. Cogn. Affect. Behav. Neurosci. 10, 141-156. doi: 10.3758/CABN.10.1.141

Eysenck, M. W., and Derakshan, N. (2011). New perspectives in attentional control theory. Pers. Individ. Differ. 50, 955-960. doi: 10.1016/j.paid.2010.08.019

Eysenck, M. W., Derakshan, N., Santos, R., and Calvo, M. G. (2007). Anxiety and cognitive performance: attentional control theory. Emotion 7, 336-353. doi: 10.1037/1528-3542.7.2.336

First, M. B., Spitzer, R. L., Gibbon, M., and Williams, J. B. W. (1997). Structured Clinical Interview for
DSM-IV Axis I Disorders: NonPatient Edition (SCID-I/NP, Version 2.0, 4/97 revision). New York, NY: Biometrics Research, New York State Psychiatric Institute.

Fonov, V. S., Evans, A. C., McKinstry, R. C., Almli, C. R., and Collins, D. L. (2009). Unbiased nonlinear average age-appropriate brain templates from birth to adulthood. Neuroimage 47:S102. doi: 10.1016/s1053-8119(09)70884-5

Fox, M. D., Buckner, R. L., White, M. P., Greicius, M. D., and PascualLeone, A. (2012). Efficacy of transcranial magnetic stimulation targets for depression is related to intrinsic functional connectivity with the subgenual cingulate. Biol. Psychiatry 72, 595-603. doi: 10.1016/j.biopsych. 2012.04.028

Friedman, N. P., and Miyake, A. (2004). The relations among inhibition and interference control functions: a latent-variable analysis. J. Exp. Psychol. Gen. 133, 101-135. doi: 10.1037/0096-3445.133.1.101

Friedman, N. P., Miyake, A., Young, S. E., DeFries, J. C., Corley, R. P., and Hewitt, J. K. (2008). Individual differences in executive functions are almost entirely genetic in origin. J. Exp. Psychol. Gen. 137, 201-225. doi: 10.1037/0096-3445. 137.2.201

Guy, S. C., Isquith, P. K., and Gioia, G. A. (2004). Behavior Rating Inventory of Executive Function - Self-report Version. Lutz, FL: Psychological Assessment Resources.

Hampshire, A., Chamberlain, S. R., Monti, M. M., Duncan, J., and Owen, A. M. (2010). The role of right inferior frontal gyrus: inhibition and attentional control. Neuroimage 50, 1313-1319. doi: 10.1016/j.neuroimage.2009.12.109.

Heller, W., Nitschke, J. B., Etienne, M. A., and Miller, G. A. (1997). Patterns of regional brain activity differentiate types of anxiety. J. Abnorm. Psychol. 106, 376-385. doi: 10.1037/0021-843X. 106.3.376

Henry, J. D., and Crawford, J. R. (2005). A meta-analytic review of verbal fluency deficits in depression. J. Clin. Exp. Neuropsychol. 27, 78-101. doi: $10.1080 / 138033990513654$

Hertel, P. T. (1997). On the contributions of deficient cognitive control to memory impairments in depression. Cogn. Emot. 11, 569-583. doi: 10.1080/026999397379890a

Herrington, J. D., Heller, W., Mohanty, A., Engels, A., Banich, M. T., Webb, A. W., et al. (2010). Localization of asymmetric brain function in emotion and depression. Psychophysiology 47, 442-454. doi: 10.1111/j.1469-8986.2009.00958.x

Herrington, J. D., Mohanty, A., Koven, N. S., Fisher, J. E., Stewart, J. L., Banich, M. T., et al. (2005). Emotion-modulated performance and activity in left dorsolateral prefrontal cortex. Emotion 5, 200-207. doi: 10.1037/1528-3542.5.2.200

Jenkinson, M. (2004). "Improving the registration of B0-disorted EPI images using calculated cost function weights," in Paper presented at Tenth International Conference on Functional Mapping of the Human Brain. (Budapest).

Jenkinson, M., Bannister, P., Brady, M., and Smith, S. (2002). Improved optimization for the robust and accurate linear registration and motion correction of brain images. Neuroimage 17, 825-841. doi: 10.1006/nimg.2002.1132

Jezzard, P., and Balaban, R. S. (1995). Correction for geometric distortion in echo planar images from B0 field variations. Magn Reson. Med. 34, 65-73. doi: 10.1002/mrm.1910340111

Jonides, J., and Nee, D. E. (2006). Brain mechanisms of proactive interference in working memory. Neuroscience 139, 181-193. doi: 10.1016/j.neuroscience.2005.06.042

Joormann, J. (2010). Cognitive inhibition and emotion regulation in depression. Curr. Dir. Psychol. Sci. 19, 161-166. doi: 10.1177/ 0963721410370293

Joormann, J., and Gotlib, I. H. (2010), Emotion regulation in depression: relation to cognitive inhibition Cogn. Emot. 24, 281-298. doi: $10.1080 / 02699930903407948$

Joormann, J., Yoon, K. L., and Zetshe, U. (2007). Cognitive inhibition in depression. Appl. Prevent. Psychol. 12, 128-139. doi: 10.1016/j.appsy.2007.09.002

Kane, M. J., and Engle, R. W. (2002). The role of prefrontal cortex in working-memory capacity, executive attention, and general fluid intelligence: An individual-differences perspective. Psychon. Bull. Rev. 9, 637-671. doi: 10.3758/BF03196323

Keller, J., Nitschke, J. B., Bhargava, T., Deldin, P., Gergen, J. A., and Miller, G. A. (2000). Neuropsychological differentiation of depression and anxiety. J. Abnorm. Psychol. 109, 3-10. doi: 10.1037/0021-843X 109.1.3

Levin, R. L., Heller, W., Mohanty, A., Herrington, J. D., and Miller, G. A. (2007). Cognitive deficits in depression and functional specificity of regional brain activity. Cogn. Ther. Res. 31, 211-233. doi: 10.1007/s10608-007-9128-z

Mayberg, H. S. (1997). Limbic-cortical dysregulation: A proposed model of depression. J. Neuropsychiatry Clin. Neurosci. 9, 471-481.

Mayberg, H. S., Liotti, S. K., Brannan, L. M., McGinnis, S., Mahurin, R. K., Jerabek, P. A., et al. (1999). Reciprocal limbic-cortical function and negative mood: converging PET findings in depression and normal sadness. Am. J. Psychiatry 156, 675-682.

Milham, M. P., and Banich, M. T. (2005). Anterior cingulate cortex: an fMRI analysis of conflict specificity and functional differentiation. Hum. Brain Mapp. 25, 328-335. doi: 10.1002/hbm.20110

Milham, M. P., Banich, M. T., and Barad, V. (2003). Competition for priority in processing increases prefrontal cortex's involvement in top-down control: an event-related fMRI study of the Stroop task. Cogn. Brain Res. 17, 212-222. doi: 10.1016/S0926-6410(03)00108-3

Miller, G. A., Crocker, L. D., Spielberg, J. M., Infantolino, Z. P, and Heller, W. (2013). Issues in localization of brain function: the case of lateralized frontal cortex in cognition, emotion, and psychopathology. Front. Integr. Neurosci. 7:2. doi: 10.3389/fnint.2013.00002

Miyake, A., Friedman, N. P., Emerson, M. J., Witzki, A. H., Howerter, A., and Wager, T. D. (2000). The unity and diversity of executive functions and their contributions to complex "frontal lobe" tasks: a latent variable analysis. Cogn. Psychol. 41, 49-100. doi: 10.1006/cogp.1999.0734

Molina, S., and Borkovec, T. D. (1994). "The Penn State Worry Questionnaire: psychometric properties and associated characteristics," in Worrying: Perspectives on Theory, Assessment and Treatment, eds G. C. L. Davey and F. Tallis (Chichester, England: Wiley), 265-283.

Moritz, S., Birkner, C., Kloss, M., Jacobsen, D., Fricke, S., and Bothern, A. (2001). Impact of comorbid depressive symptoms on neuropsychological performance in obsessive-compulsive disorder. J. Abnorm. Psychol. 110, 653-658. doi: 10.1037/0021-843X.110.4.653

Nelson, J. K., Reuter-Lorenz, P. A., Sylvester, C.-Y. C, Jonides, J., and Smith, E. E. (2003) Dissociable neural mechanisms underlying response-based and familiarity-based conflict in working memory. Proc. Natl. Acad. 
Sci. U.S.A. 100, 11171-11175. doi: 10.1073/pnas. 1334125100

Nitschke, J. B., Heller, W., Imig, J. C., McDonald, R. P., and Miller, G. A. (2001). Distinguishing dimensions of anxiety and depression. Cogn. Ther. Res. 25, 1-22. doi: 10.1023/A:1026485530405.

Nitschke, J. B., Heller, W., Palmieri, P. A., and Miller G. A. (1999). Contrasting patterns of brain activity in anxious apprehension and anxious arousal. Psychophysiology 36, 628-637. doi: $10.1017 /$ S0048577299972013.

Pizzagalli, D. A., Peccoralo, L. A., Davidson, R. J., and Cohen, J. D. (2006). Resting anterior cingulate activity and abnormal responses to errors in subjects with elevated depressive symptoms: a 128channel EEG study. Hum. Brain Mapp. 27, 185-201. doi: 10.1002/ hbm. 20172

Rogers, M. A., Kasai, K., Koji, M., Fukuda, R., Iwanami, A., Nakagome, K., et al. (2004). Executive and prefrontal dysfunction in unipolar depression: a review of neuropsychological and imaging evidence. Neurosci. Res. 50, 1-11. doi: 10.1016/j.neures.2004. 05.003

Siemer, M. (2005). Mood congruent cognitions constitute mood experience. Emotion 5, 296-308. doi: 10.1037/1528-3542.5.3.296

Silton, R. L., Heller, W., Towers, D. N., Engels, A. S., Edgar, J. C., Spielberg, J. M., et al. (2011). Depression and anxiety distinguish frontocingulate cortical activity during top-down attentional control. J. Abnorm.
Psychol. 120, 272-285. doi: 10.1037/ a0023204

Silton, R. L., Miller, G. A., Towers, D. N., Engels, A. S., Edgar, J. C., Spielberg, J. M., et al. (2010). The time course of activity in dorsolateral prefrontal cortex and anterior cingulate cortex during top-down attentional control. Neuroimage 50, 1292-1302. doi: 10.1016/j.neuroimage.2009. 12.061

Snyder, H. R. (2013). Major depressive disorder is associated with broad impairments on neuropsychological measures of executive function: a meta-analysis and review. Psychol. Bull. 139, 81-132. doi: 10.1037/ a0028727

Spielberg, J. M., Miller, G. A., Engels, A. S., Herrington, J. D., Sutton, B. P., Banich, M. T., and Heller, W. (2011). Trait approach and avoidance motivation: lateralized neural activity associated with executive function. Neuroimage 54, 661-670. doi: $\quad 10.1016 / j$.neuroimage.2010. 08.037

Stroop, J. R. (1935). Studies of interference in serial verbal reactions. J. Exp. Psychol. 18, 643-662. doi: 10.1037/h0054651

Wager, T. D., and Smith, E. E. (2003). Neuroimaging studies of working memory: a meta-analysis. Cogn. Affect. Behav. Neurosci. 3, 255-274. doi: 10.3758/CABN.3.4.255

Ward, D. B. (2000). Simultaneous inference for fMRI data. Retrieved from: http://afni.nimh.nih.gov./ pub/dist/doc/manual/AlphaSim.pdf (Accessed July 27, 2006). doi: 10.1111/ejn.12083
Warren, S. L., Miller, G. A., and Heller, W. (2008). "Emotional facilitation and disruption of memory," in Human Learning: Biology, Brain and Neuroscience, eds M. Guadagnoli, A. S. Benjamin, S. de Belle, B. Etnyre, and T. Polk (Holland: Elsevier).

Watson, D., Clark, L. A., Weber, K., Assenheimer, J. S., Strauss, M. E., and McCormick, R. A. (1995a). Testing a tripartite model: II. Exploring the symptom structure of anxiety and depression in student, adult, and patient samples. J. Abnorm. Psychol. 104, 15-25. doi: 10.1037/0021-843X.104. 1.15

Watson, D., Weber, K., Assenheimer, J. S., Clark, L. A., Strauss, M. E., and McCormick, R. A. (1995b). Testing a tripartite model: I. Evaluating the convergent and discriminant validity of anxiety and depression symptom scales. J. Abnorm. Psychol. 104, 3-14. doi: 10.1037/0021-843X. 104.1.3

Weiland-Fiedler, P., Erickson, K., Waldeck, T., Luckenbaugh, D. A., Pike, D., Bonne, O. et al. (2004). Evidence for continuing neuropsychological impairments in depression. J. Affect. Disord. 82, 253-258. doi: 10.1016/j.jad.2003.10.009

Woolrich, M. W., Ripley, B. D. Brady, M., and Smith, S. M. (2001). Temporal autocorrelation in univariate linear modeling of fMRI data. Neuroimage 14, 1370-1386. doi: 10.1006/nimg. 2001.0931
Yee, C. M., and Miller, G. A. (1994). A dual-task analysis of resource allocation in dysthymia and anhedonia. J. Abnorm. Psychol. 103, 625-636. doi: $\quad 10.1037 / 0021-843 X .103$ 4.625

Zelazo, P. D., and Cunningham, W. A. (2007). "Executive function: mechanisms underlying emotion regulation," in Handbook of Emotion Regulation, ed J. J. Gross (New York, NY: Guilford Press), 135-158.

Conflict of Interest Statement: The authors declare that the research was conducted in the absence of any commercial or financial relationships that could be construed as a potential conflict of interest.

Received: 14 February 2013; accepted: 25 May 2013; published online: 13 June 2013.

Citation: Warren SL, Crocker LD, Spielberg JM, Engels AS, Banich MT, Sutton BP, Miller GA and Heller $W$ (2013) Cortical organization of inhibition-related functions and modulation by psychopathology. Front. Hum. Neurosci. 7:271. doi: 10.3389/fnhum. 2013.00271

Copyright (c) 2013 Warren, Crocker, Spielberg, Engels, Banich, Sutton, Miller and Heller. This is an open-access article distributed under the terms of the Creative Commons Attribution License, which permits use, distribution and reproduction in other forums, provided the original authors and source are credited and subject to any copyright notices concerning any third-party graphics etc. 